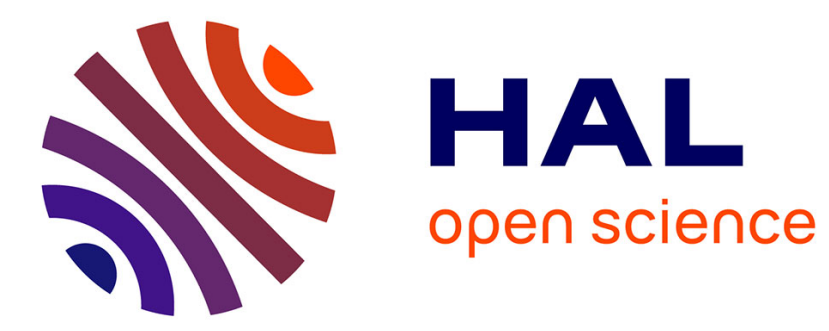

\title{
A geometrical framework for the statics of materials with microstructure
}

Reuven Segev

\section{To cite this version:}

Reuven Segev. A geometrical framework for the statics of materials with microstructure. Mathematical Models and Methods in Applied Sciences, 1994, 4 (6), 27 p. hal-00956474

\section{HAL Id: hal-00956474 \\ https://hal.science/hal-00956474}

Submitted on 7 Mar 2014

HAL is a multi-disciplinary open access archive for the deposit and dissemination of scientific research documents, whether they are published or not. The documents may come from teaching and research institutions in France or abroad, or from public or private research centers.
L'archive ouverte pluridisciplinaire HAL, est destinée au dépôt et à la diffusion de documents scientifiques de niveau recherche, publiés ou non, émanant des établissements d'enseignement et de recherche français ou étrangers, des laboratoires publics ou privés. 


\title{
A GEOMETRICAL FRAMEWORK FOR THE STATICS OF MATERIALS WITH MICROSTRUCTURE
}

\author{
REUVEN SEGEV \\ Department of Mechanical Engineering, Ben-Gurion University, \\ P. O. Box 653, Beer-Sheva 84105 , Israel
}

Communicated by K. R. Rajagopal

\begin{abstract}
A geometrical framework for the formulations of the invariant mechanical theories of materials with microstructure is presented. The suggested framework is based on the construction of an infinite-dimensional manifold containing the configurations of the body. Each configuration includes both the macro- and the micro-states of the body. The notions of forces, stresses, balance laws and latent microstructure are discussed. Liquid crystals are used as an example.
\end{abstract}

\section{Introduction}

While in the usual formulation of continuum mechanics the states of the material points are described by the places that they occupy in three-dimensional Euclidean space, in various theories of bodies with microstructure additional parameters are needed in order to specify the state of the material points. These additional parameters are sometimes referred to as "internal degrees of freedom" defining the "microstates." In some of the examples of theories of bodies with microstructure, ${ }^{2,7}$ the microstates are specified by points on a differentiable manifold rather than the restricted case of a Euclidean space. Thus, it is natural to study the mechanics of bodies for which the states of the material points take values on a general differentiable manifold. This is indeed the setting of the framework for the mechanics of bodies with microstructure suggested by Capriz. ${ }^{2}$ Formulations of some notions of continuum mechanics in the case where both the body and space are modeled by differentiable manifolds were given in previous works. ${ }^{5,11}$ The basic notion used for the formulation of statics in these works is the configuration manifold containing all the possible configuration of the body. Such a configuration manifold is naturally infinite-dimensional and its properties reflect the kinematics of the body. Then, virtual displacements or generalized velocities are defined as tangent vectors to the configuration manifold and forces are defined as linear forms on the infinitedimensional spaces of virtual displacements. In this way the properties of forces 
are induced by the kinematics of the system. In particular, stress theory follows mathematically from the basic definitions.

This paper uses some of the constructions of the works mentioned, applies them to the case of materials with microstructure and extends some of them. Particular attention is given to the study of a symmetry group action on the manifold of microstates. It is within the framework of the geometrical structure of a group action that notions such as resultants or totals and balance laws may be defined. Our construction should be compared to a recent work by Capriz and Virga. ${ }^{3}$ An additional notion that we study from a generalized point of view is that of a body with latent microstructure. ${ }^{1}$

The fact that the microstates are valued on a differentiable manifold introduces a few difficulties in the formulation of the theory in comparison with the formulation of the mechanics of simple bodies. These difficulties stem from the lack of geometrical structure available in the Euclidean space and we will illustrate them informally in the following section. The theory of liquid crystals will serve as a standard example for the applications of notions introduced.

\section{Generalized Mixtures-An Example}

Consider a mixture that occupies a fixed region $B$ in the Euclidean space. The mixture comprises $n$ constituents. Each of the constituents occupies the whole region $B$, and $c^{p}$ denotes the concentration of the $p$ th constituent. The concentrations are positive real numbers so that the microstates at each point of the mixture is described by the collection $\left(c^{1}, c^{2}, \ldots, c^{n}\right) \in\left(\mathbb{R}^{+}\right)^{n}$. Note that the concentrations $c^{p}$ are just measured quantities and need not have intrinsic physical meaning. For example, the various constituents may have different colors and the $c^{p}$ values could be the intensities of these colors. It is clear that other means of measuring the concentrations of the constituents are valid, so the microstates of the system could be specified by other coordinates or internal degrees of freedom. For example, say $\left(b^{1}, b^{2}, \ldots, b^{n}\right) \in\left(\mathbb{R}^{+}\right)^{n}$ are other coordinates on the manifold of microstates and the coordinate transformations (e.g. $b^{r}\left(c^{p}\right)$ ) between the two coordinate systems are differentiable and nonsingular.

Examples for similar mixtures that appear in the literature are the continua with voids, liquid with bubbles, dilatant granular material discussed by Capriz ${ }^{2}$ and the mutliphase mixtures discussed by Passman. ${ }^{10}$ In all these examples the microstate of a generic body point may be specified by the volume fractions of the various constituents (or voids), but other parameters, related to them nonlinearly, may serve as well.

One will tend to define a micro-generalized-velocity or a micro-virtualdisplacement of the point $X$ in the mixture using the $c^{p}$ coordinates by $\delta c^{p}$ and the corresponding velocity is specified using the $b^{r}$ coordinates as $\delta b^{r}=b_{, p}^{r} \delta c^{p}$. Here, the comma followed by a $p$ denotes partial differentiation of the coordinate transformation with respect to the $c^{p}$ coordinate. 
A difficulty in assigning a physical meaning to the collection $\left(\delta c^{p}\right)$ may be observed: A single collection $\left(\delta c^{p}\right)$ will result in two different collections $\left(\delta b^{r}\right)$ when the micro-virtual displacements are at two distinct microstates $\left(c_{1}^{p}\right),\left(c_{2}^{p}\right)$. Because of the nonlinear character of the coordinate transformations, the partial derivatives $b_{, p}^{r}$ in the transformation rule for the virtual displacements will be different. A micro-virtual-displacement or a generalized velocity may be defined as a tangent to the manifold of microstates as will be done later.

Even if virtual displacements are defined invariantly we do not have in general an invariant way to construct an inner product between virtual displacements. Such an inner product is essential if we wish to define micro-forces as elements of inner product spaces as suggested by Noll, ${ }^{8}$ Truesdell ${ }^{14}$ and others. The inner product between a micro-force and a micro-generalized velocity to evaluate the power cannot be invariantly performed. A force may be defined as a covector producing the virtual power from the virtual displacement.

Without additional structure, it will not make sense to compare two virtual displacements at two distinct microstates or to translate a virtual displacement from one microstate to another. The same operations cannot be defined for forces as their properties are derived from those pertaining to generalized velocities. It follows that the force acting on a material point having a certain state cannot be added to the force acting on a second material point having another microstate. Thus, it will not be possible to integrate the force in order to obtain totals or resultants as attempted by Capriz and Virga ${ }^{3}$ following the general framework of Noll $^{8}$ and Truesdell. ${ }^{14}$

The micro-configuration of the mixture will be specified by the map $c^{p}=c^{p}\left(X^{I}\right)$ if we use the $c^{p}$ coordinates and by mappings of the form $b^{r}=b^{r}\left(X^{I}\right)$ if we use the $b^{r}$ coordinates ( $X^{I}$ denote the coordinates for the body points). The configuration gradients with respect to the two coordinates systems will be related by $b_{, I}^{r}=b_{, p}^{r} c_{, I}^{p}$.

Various authors (e.g., Capriz ${ }^{2}$ and Passman et al. ${ }^{10}$ ) obtain the micro-self-force as the derivative of a potential energy function $u$ with respect to the microstate and the micro-stress is obtained as the derivative of the potential energy function with respect to the gradient of the micro-configuration. The relations between the derivatives of the potential energy functions when using the two different coordinate systems $c^{p}$ and $b^{r}$ are as follows:

$$
\begin{aligned}
\frac{\partial u}{\partial b^{r}} & =\frac{\partial u}{\partial c^{p}} \frac{\partial c^{p}}{\partial b^{r}}+\frac{\partial u}{\partial c_{, I}^{p}} \frac{\partial}{\partial X^{I}}\left(\frac{\partial c^{p}}{\partial b^{r}}\right), \\
\frac{\partial u}{\partial b_{, I}^{r}} & =b_{, p}^{r} \frac{\partial u}{\partial c_{, I}^{p}} .
\end{aligned}
$$

Hence, the micro-self-force cannot be separated invariantly from the micro-stress without additional structure. 
In the following sections we introduce the geometrical structure needed in order to overcome the various difficulties described.

\section{The Geometrical Objects Associated with the Statics of Materials with Microstructure}

In order to describe statics we consider a typical slice of the event space (or spacetime). In accordance with traditional mechanics we assume that this collection of simultaneous events is a Euclidean space $E$ and we will denote its tangent (or translation) space by $V$. A differentiable manifold $M$ will model the collection of values that the microstates of the material points may assume. It will be assumed that $M$ does not have a boundary. The body will be modeled by a compact threedimensional submanifold with a boundary $B$ of $E$.

\section{Example: The manifold of microstates for a liquid crystal}

In the continuum theory of liquid crystals the microstates of the material points describe the lines in space along which the molecules are located. We identify the physical space with a particular three-dimensional Euclidean space. Hence, for a particular position in space $x$ of the center of the molecule, the collection of the microstates of that particular molecule is the collection of all lines passing through $x$. Because of the parallelism of the Euclidean space we may identify the collection of lines passing through a point $x$ in space with the collection of lines passing through any other point $y$. Thus, we may say that the microstates of the material points are valued in the set $P^{2}$ of lines passing through a generic point $O$ in the Euclidean space. The set $P^{2}$ is traditionally called the projective plane and its properties will be reflected in the micromechanics of liquid crystals.

The projective plane $P^{2}$ is one of the classical examples of a non-trivial differentiable manifolds. It is a two-dimensional manifold that cannot be covered by a single chart and that cannot be embedded in $\mathbb{R}^{3}$. The projective plane may be described alternatively using Fig. 1 depicting a unit sphere in the Euclidean space centered at $O$ and a Euclidean plane tangent to it. Each line through $O$ intersects the sphere at two diametrically opposite points $x, x^{\prime}$, and any two such points on the sphere determine a unique line through $O$. It follows that we can define $P^{2}$ as the quotient set obtained from the unit sphere centered at $O$ by the equivalence relation that identifies two points $x, x^{\prime}$ if they are diametrically opposite. We also note that any line that is not parallel to the tangent Euclidean plane intersects it at some point $y$ and can be identified with that point. Lines parallel to the plane may be identified with imaginary points at infinity.

In order to define the manifold structure on $P^{2}$ we first choose a Cartesian coordinate system originating at the point $O$ so that the coordinates of a point $x$ in space will be $x^{1}, x^{2}, x^{3}$. Consider the Euclidean plane $x^{3}=-1$ and let $U_{1}$ denote the collection of lines that intersect it. With each line we may associate a pair of 


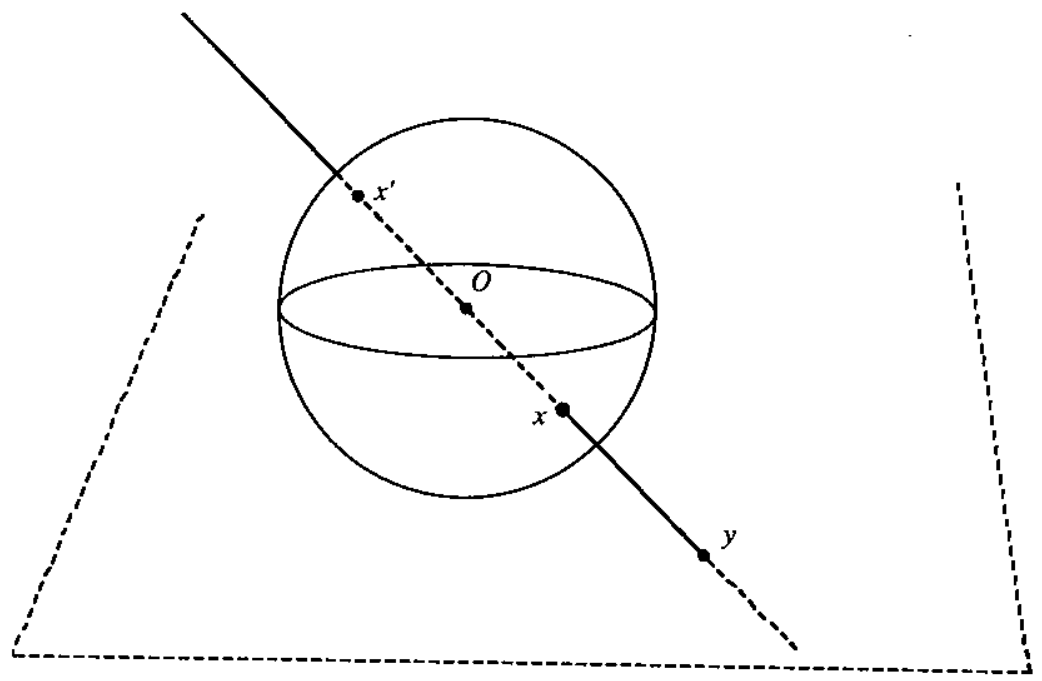

Fig. 1. The projective plane.

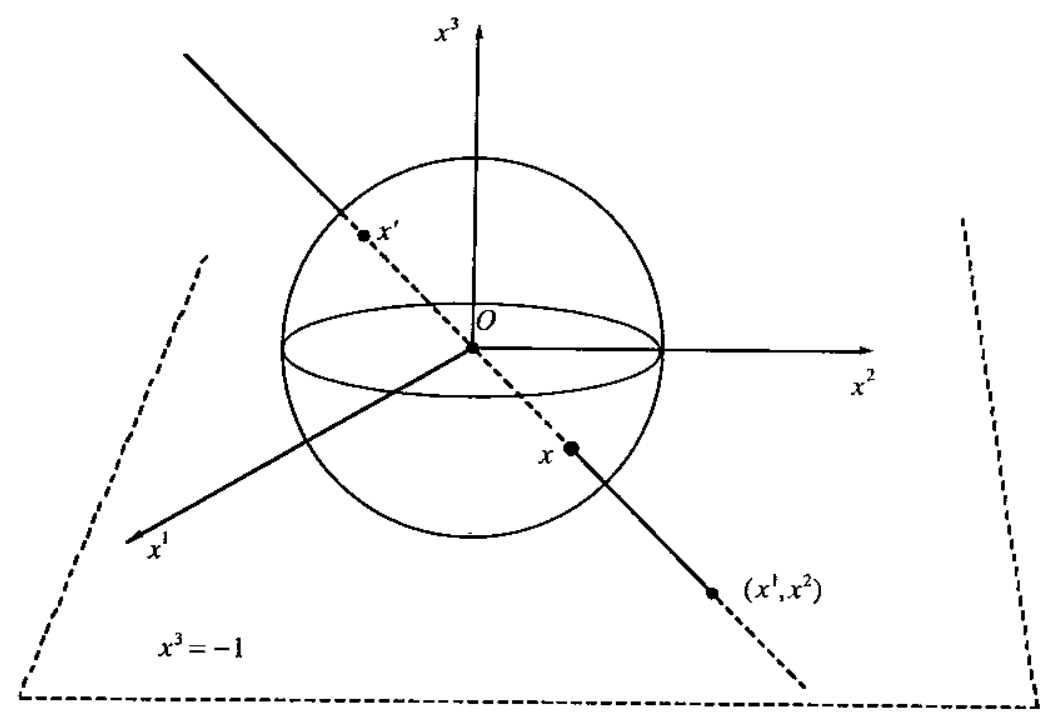

Fig. 2. A chart on the projective plane.

numbers, $\left(x^{1}, x^{2}\right)$, the first two coordinates of the point of intersection of the line with the plane (see Fig. 2). Now we may consider the plane $x^{2}=-1$ and the set of lines $U_{2}$ that intersect it. Again we can associate with each line in $U_{2}$ the pair of coordinates $\left(x^{1}, x^{3}\right)$ of the point of its intersection with the plane. Lastly, $U_{3}$ is the collection of lines that intersect the $x^{1}=-1$ plane and with each line in $U_{3}$ we associate the coordinates $\left(x^{2}, x^{3}\right)$. Clearly, any line intersects 
at least one of the planes mentioned and it follows that the sets $U_{1}, U_{2}, U_{3}$ cover $P^{2}$. It can be shown that for the lines that belong to more than one of the sets $U_{1}, U_{2}, U_{3}$ the transformations of coordinates are differentiable and nonsingular. Thus, $P^{2}$ may be given the structure of a two-dimensional manifold and in the sequel we will use $\left(n^{1}, n^{2}\right)$ to denote the coordinates of an element $n$ of the projective plane in a chart whose domain contains $n$.

Denote by $p r: V \rightarrow P^{2}$ the mapping that associates with any vector in the tangent space $V$ the line through $O$ on which it is located. Using the charts we defined above, this mapping gives two out of the three coordinates and is clearly differentiable.

A virtual motion of a molecule of liquid crystal is a mapping $m: \mathbb{R} \rightarrow P^{2}$. Here, we identify the time axis with $\mathbb{R}$. A generalized velocity, or a microvelocity, of the molecule at the microstate $n$ is a rate of change of a motion when it is passing through $n$. Mathematically, the velocity is a tangent vector to the configuration space $P^{2}$ at the configuration $n$, i.e. it is a collection of motions of the molecule, that have the same derivative at $n$ when we use a chart in a neighborhood of $n$ to describe the motions by coordinates. Since the motion is represented in a neighborhood of $n$ by the two functions $n^{1}(t), n^{2}(t)$ ( $t$ represents the time variable), the chart may be used to represent the tangent vector, or the velocity by the time derivatives $\dot{n}^{1}, \dot{n}^{2}$, evaluated at the time $t_{0}$ in which the motion passes through $n$. The tangent space to the projective plane at $n, T_{n} P^{2}$ is a two-dimensional vector space and may be embedded in $\mathbb{R}^{3}$. A natural embedding of $T_{n} P^{2}$ in $V$ (the tangent space of the Euclidean space) can be obtained as follows. Consider a rotating line $m(t)$ with $m\left(t_{0}\right)=n$.

Let $p$ and $p^{\prime}$ be the points on that line that have a unit distance from $O$ and let $\mathbf{v}$ and $\mathbf{v}^{\prime}$ be the velocities of these points at time $t_{0}$ (see Fig. 3). These velocities are clearly perpendicular to the line $n$ and satisfy $\mathbf{v}=-\mathbf{v}^{\prime}$. It is clear that these velocities determine the tangent to the motion of the line at $n$ uniquely. Both $\mathbf{v}$ and $\mathbf{v}^{\prime}$ may be specified by a unique vector $\omega \in V$ in the plane through $O$ which is perpendicular to $n$. Consider the vectors $\overline{O p}$ and $\overline{O p^{\prime}}$ along $n$. Since the vectors $\mathbf{v}$ and $\mathbf{v}^{\prime}$ are perpendicular to $\overline{O p}$ and $\overline{O p^{\prime}}$, respectively, there is a vector $\boldsymbol{\omega}$ such that

$$
\mathbf{v}=\boldsymbol{\omega} \times \overline{O p} \quad \text { and } \quad \mathbf{v}^{\prime}=\boldsymbol{\omega} \times \overline{O p^{\prime}}
$$

where $\times$ denotes the vector product. It is clear that $\omega$ is perpendicular to the velocities as well as to the line $n$. As each such vector $\boldsymbol{\omega}$ determines a unique pair of velocities $\mathbf{v}$ and $\mathbf{v}^{\prime}$, the identification of the micro-velocity with the vector $\boldsymbol{\omega}$ is complete. It is natural to refer to $\boldsymbol{\omega}$ as the angular velocity of the molecule associated with the micro-velocity $v$. We conclude that we may identify the tangent space $T_{n} P^{2}$ with the plane in $V$ perpendicular to $n$. The tangent bundle of the projective plane, i.e. the collection of all micro-velocities corresponding to all the microstates will be denoted by $T P^{2}$ and $\tau: T P^{2} \rightarrow P^{2}$ 


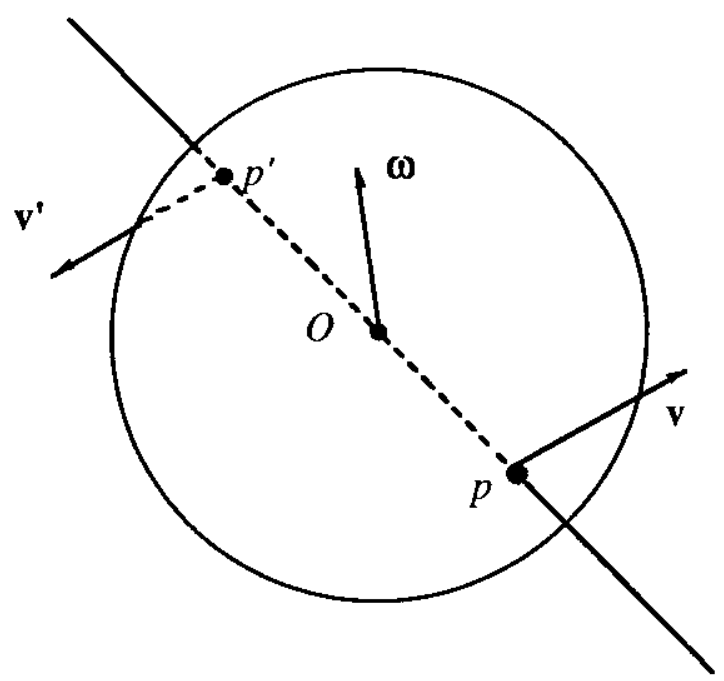

Fig. 3. Micro-velocity of a point in a liquid crystal.

will denote the natural projection assigning to each tangent vector the point to which it is attached.

A geometrical configuration is a continuously differentiable embedding of the body in E. A micro-configuration is a continuously differentiable mapping of the body into the manifold of microstates. A physical configuration is a differentiable mapping of the body into $E \times M$ such that its first component is a geometrical configuration. That is, $\kappa: B \rightarrow E \times M$ is a physical configuration if $p r_{1} \circ \kappa: B \rightarrow E$ is an embedding. In the last expression, $p r_{1}: E \times M \rightarrow E$ is the first natural projection of the Cartesian product and $p r_{2}$ is defined similarly. Clearly, a physical configuration is an embedding.

We will denote the coordinates of a point $X \in B$ under a local chart by $X^{I}$, the coordinates of a point $x \in E$ by $x^{i}$ and the coordinates of a microstate $\nu \in M$ by $\nu^{p}$. Thus, a physical configuration will be represented locally by mappings $X^{I} \mapsto\left(x^{i}\left(X^{I}\right), \nu^{p}\left(X^{I}\right)\right)$.

The collection of all physical configurations will be denoted by $Q$ and will be referred to as the configuration space. The collection of geometrical configurations will be denoted by $Q_{1}$ and the collection of all micro-configurations will be denoted by $Q_{2}$. Since any pair $\left(\kappa_{1}, \kappa_{2}\right), \kappa_{1} \in Q_{1}, \kappa_{2} \in Q_{2}$, determines a physical configuration we have $Q=Q_{1} \times Q_{2}$.

In order to apply the basic geometrical framework, consisting of a configuration manifold, its tangent bundle containing the virtual displacements (or generalized velocities) and the cotangent bundle containing the (generalized) forces, to the statics of materials with microstructure, we have to specify a manifold structure for the configuration space $Q$. The basic results concerning manifolds of mappings 
that we will use later can be found in the works of Eliasson, ${ }^{4} \mathrm{Hirsch}^{6}$ and Palais. ${ }^{9}$ These results are briefly reviewed in the next section.

\section{Review of the Basic Results on Manifolds of Mappings}

Consider a manifold without a boundary $S$ and a compact manifold $R$ and denote by $C^{1}(R, S)$ the collection of continuously differentiable mappings $R \rightarrow S$. (For example, $C^{1}(B, M)=Q_{2}$.) In constructing a manifold structure for $C^{1}(R, S)$ it is convenient to use the pullback $h^{*} \tau_{S}: h^{*} T_{S} \rightarrow R$ of the tangent bundle $\tau_{S}: T_{S} \rightarrow S$ by a mapping $h \in C^{1}(R, S)$, which is a vector bundle over $R$. For each $r \in R$, the fiber $\left(h^{*} T_{S}\right)_{r}$ is the tangent space $T_{h(r)} S$ (the tangent space to $S$ at $h(r)$ ). A section $u$ of $h^{*} \tau_{S}: h^{*} T_{S} \rightarrow R$ is a mapping $u: R \rightarrow h^{*} T_{S}$ such that $h^{*} \tau_{S} \circ u=i d_{R}$. We will denote the collection of differentiable sections of $h^{*} \tau_{S}: h^{*} T_{S} \rightarrow R$ by $C^{1}\left(h^{*} \tau_{S}\right)$. A section $u \in C^{1}\left(h^{*} \tau_{S}\right)$ may be identified with a mapping $\hat{u}: R \rightarrow T S$ satisfying $\tau_{S} \circ \hat{u}=h$. The vector field $\hat{u}$ is defined by $\hat{u}(r)=u(r) \in T_{h(r)} S$ and the identification is possible by definition, $\left(h^{*} T_{S}\right)_{r}$ is the tangent space $T_{h(r)} S$. A mapping $\hat{u}: R \rightarrow T S$ satisfying $\tau_{S} \circ \hat{u}=h$, will be referred to as a vector field along $h$ and may be conceived as an infinitesimal variation of $h$. If $h$ is any mapping $R \rightarrow S$, a vector field $\hat{u}: R \rightarrow T S$ along $h$ induces a section $u \in C^{1}\left(h^{*} \tau_{S}\right)$ by the same identification. In the case where $h$ is an embedding, $h^{*} \tau_{S}: h^{*} T_{S} \rightarrow R$ may be identified with the restriction of $T S$ to $h(R)$. A section $u: S \rightarrow T S$ induces naturally a section $u \circ h$ of the vector bundle $h^{*} \tau_{M}$. We will use the notation $h^{*}$ for the mapping $C^{1}\left(\tau_{S}\right) \rightarrow C^{1}\left(h^{*} \tau_{M}\right)$ such that $h^{*}(u)=h \circ u$.

Denoting by $r^{i}$ local coordinates in $R$ and denoting by $\left(s^{p}, b^{q}\right)$ the coordinates in a natural chart in $T S$, the coordinates in a local natural chart in $h^{*} T_{S}$ will be of the form $\left(r^{i}, b^{p}\right)$. A section $u$ of the pullback will be locally of the form $r^{i} \mapsto\left(r^{i}, b^{p}\left(r^{i}\right)\right)$.

The vector space $C^{1}\left(h^{*} \tau_{S}\right)$ like any other vector space of $C^{1}$ sections of a vector bundle can be endowed with the structure of a Banachable space using the $C^{1}$ norm in the local charts. The collection of mappings $C^{1}(R, S)$ can be given a structure of a Banach manifold and the tangent space at any mapping $h \in C^{1}(R, S)$ can be identified with the Banachable space $C^{1}\left(h^{*} \tau_{S}\right)$. The identification of $C^{1}\left(h^{*} \tau_{S}\right)$ with the collection of differentiable vector fields along $h$ implies that we can identify tangent vectors in $T_{h} C^{1}(R, S)$ with vector fields along $h$. In the context of kinematics, these vector fields are interpreted as fields of generalized velocity or virtual displacements. The collection of embeddings of $R$ in $S$ is an open subset of $C^{1}(R, S)$ if we use the $C^{1}$ topology. It follows that the tangent space to the manifold of embeddings at $h$ is again $C^{1}\left(h^{*} \tau_{S}\right)$.

The jet bundle $J\left(h^{*} \tau_{S}\right): J\left(h^{*} T_{S}\right) \rightarrow R$ is the fiber bundle over $R$ whose fiber at $r \in R$ is the collection of values that a first order Taylor expansion of sections of $h^{*} \tau_{S}$ at $r$ may have. Thus, if $\operatorname{dim}(R)=m$ and $\operatorname{dim}(S)=n$, at any $r \in R$ the fiber $J\left(h^{*} T_{S}\right)_{r}$ is isomorphic to $\mathbb{R}^{n} \times L\left(\mathbb{R}^{m}, \mathbb{R}^{n}\right)$. The jet extension mapping

$$
j: C^{1}\left(h^{*} \tau_{S}\right) \rightarrow C^{0}\left(J\left(h^{*} \tau_{S}\right)\right)
$$

assigns to each section $u$ of $h^{*} \tau_{S}$ the section of the jet bundle whose value at 
any point is the first order Taylor expansion of $u$ at that point. Thus, assuming that $u$ is represented locally by $r^{i} \mapsto b^{p}\left(r^{i}\right)$, then $j(u)$ is represented locally by $r^{i} \mapsto\left(r^{i}, b^{p}\left(r^{i}\right), b_{, j}^{p}\left(r^{i}\right)\right)$. Clearly, the jet extension mapping $j: C^{1}\left(h^{*} \tau_{S}\right) \rightarrow$ $C^{0}\left(J\left(h^{*} \tau_{S}\right)\right)$ is linear and injective. Its local representatives are norm preserving by the definitions of the $C^{0}$ and $C^{1}$ norms.

All these definitions and results hold in the case of the statics of materials with microstructure for both $Q_{1}$ which is the set of embeddings of $B$ in $E$ and for $Q_{2}=C^{1}(B, M)$.

\section{The Representation of Forces by Stresses}

In this section we want to examine the most general implications of the definition of a force at the configuration $\kappa \in Q$ as a continuous linear functional defined on the space $T_{\kappa} Q$. Since $Q=Q_{1} \times Q_{2}$, at any configuration $\kappa=\left(\kappa_{1}, \kappa_{2}\right),\left(T^{*} Q\right)_{\kappa}=$ $\left(T^{*} Q_{1}\right)_{\kappa_{1}} \times\left(T^{*} Q_{2}\right)_{\kappa_{2}}$. It follows that a force $f \in T_{\kappa}^{*} Q$ is of the form $\left(f_{1}, f_{2}\right) \in$ $\left(T^{*} Q_{1}\right)_{\kappa_{1}} \times\left(T^{*} Q_{2}\right)_{\kappa_{2}}$, so that for a generalized velocity $u=\left(u_{1}, u_{2}\right) \in T_{\kappa} Q=$ $\left(T^{*} Q_{1}\right)_{\kappa_{1}} \times\left(T^{*} Q_{2}\right)_{\kappa_{2}}, f(u)=f_{1}\left(u_{1}\right)+f_{2}\left(u_{2}\right)$. Since $T Q_{1}$ is a particular case of $T Q_{2}$, it is sufficient firstly to study the properties of forces as linear functionals defined on tangent spaces of the form $T_{h} Q_{2}=C^{1}\left(h^{*} \tau_{M}\right)$. Hence, a microforce, the second component of a force, is a continuous linear mapping $f: C^{1}\left(h^{*} \tau_{M}\right) \rightarrow \mathbb{R}$. In the sequel, where no confusion can occur, we will omit the index 2 when considering objects related to the microstructure.

The basic property of forces implied by the definition above is the representation of forces by stresses. ${ }^{11}$ Since the jet extension mapping $j: C^{1}\left(h^{*} \tau_{M}\right) \rightarrow$ $C^{0}\left(J\left(h^{*} \tau_{M}\right)\right)$ is injective, linear and preserves any norm induced by local charts, it follows that any force $f$ may be represented by an element $\Sigma \in C^{0}\left(J\left(h^{*} \tau_{M}\right)\right)^{*}$ in the form $f=j^{*}(\Sigma)$. Here, $j^{*}: C^{0}\left(J\left(h^{*} \tau_{M}\right)\right)^{*} \rightarrow C^{1}\left(h^{*} \tau_{M}\right)^{*}$ is the adjoint of the jet extension mapping. That is, for every force $f$ there is an element $\Sigma \in C^{0}\left(J\left(h^{*} \tau_{M}\right)\right)^{*}$, called a stress representation of the force, such that $f=j^{*}(\Sigma)$. The definition of the adjoint implies that for a stress representation $\Sigma$ of the force $f$ we have $f=\Sigma \circ j$ so that $f(u)=\Sigma(j(u))$ for all $u \in C^{1}\left(h^{*} \tau_{M}\right)=T_{h} Q_{2}$. As a result of the fact that $j$ is not surjective, we cannot expect the representation to be unique so that two different stresses may represent the same force. The equation $f=j^{*}(\Sigma)$ is a generalized form of the equilibrium equation of continuum mechanics and the condition $f(u)=\Sigma(j(u))$ is a generalized form of the principle of virtual work in continuum mechanics.

Stresses are linear functionals on spaces of continuous sections of the vector bundle $J\left(h^{*} \tau_{M}\right)$ and as such (see previous work ${ }^{11}$ and references therein) they are measures over the body valued in the dual bundle $J\left(h^{*} \tau_{M}\right)^{*}$. Henceforth, we will refer to elements of $C^{0}\left(J\left(h^{*} \tau_{M}\right)\right)^{*}$ as stress measures. If the stress $\Sigma$ represents the force $f$ we have

$$
f(u)=\int_{B} j(u) d \Sigma, \quad \text { for all } u \in C^{1}\left(h^{*} \tau_{M}\right)=T_{h} Q_{2}
$$


If we denote by $\left(\nu^{p}, u^{q}\right)$ the coordinates in a local chart in $T M$ so that the induced local coordinates in $h^{*} \tau_{M}$ are of the form $\left(X^{I}, u^{q}\right)$, the local coordinates in $J\left(h^{*} \tau_{M}\right)$ are of the form $\left(X^{I}, u^{q}, L_{J}^{p}\right)$ and the local coordinates in its dual bundle are of the form $\left(X^{I}, s_{q}, S_{p}^{J}\right)$. The evaluation of the action $S(w), S \in J\left(h^{*} \tau_{M}\right)_{X}^{*}$, $w \in J\left(h^{*} \tau_{M}\right)_{X}$, is in the form $s_{q} u^{q}+S_{p}^{I} L_{I}^{p}$. Thus, a stress $\Sigma$ can be represented locally by measures $\left(\Sigma_{q}, \Sigma_{p}^{I}\right)$ defined over the domain of a chart. These local representatives are combined by means of a partition of unity into a global invariant element of $C^{0}\left(J\left(h^{*} \tau_{M}\right)\right)^{*}$. For the particular case where the jet bundle can be covered by one chart, the fact that the stress measure $\Sigma$ represents the force $f$, may be expressed using coordinates of the form

$$
f(u)=\int_{B} u^{q} d \Sigma_{q}+\int_{B} u_{, I}^{p} d \Sigma_{p}^{I} \text { for all } u .
$$

The definition of forces does not suggest a way to restrict a given force on a body to its sub-bodies. By a force system on the body $B$ we mean an assignment of a force $f_{P}$ to each sub-body $P \subset B$. If a stress $\Sigma$ is given, the fact that a measure may be restricted to sub-bodies, implies that $\Sigma$ induces a force system such that

$$
f_{P}(u)=\int_{P} j(u) d \Sigma
$$

for all $u$ defined on $P$. Therefore, we may say roughly that a stress measure representing a given force enables us to restrict the force to the sub-bodies. This restriction depends on $\Sigma$ which is not uniquely determined by the force. However, it can be shown ${ }^{11,13}$ that if a force system is given on the body, there is at most one stress measure that represents the various forces on the sub-bodies. In addition, conditions for a force system $\left\{f_{P}\right\}$ so that a stress $\Sigma$ representing the forces on the various sub-bodies exists, are given in the above mentioned work. ${ }^{13}$ These conditions generalize the classical Cauchy conditions in the following aspects. They hold for general manifolds, they are not related to the assumption of equilibrium or an equivalent assumption and they hold for stresses that may be as irregular as measure in accordance with the general framework presented here. The generalized Cauchy conditions are as follows:

(i) Additivity. If $P_{1}$ and $P_{2}$ are disjoint sub-bodies of $B$ then for any $u \in$ $C^{1}\left(h^{*} \tau_{M}\right)$

$$
f_{P_{1} \cup P_{2}}\left(\left.u\right|_{P_{1} \cup P_{2}}\right)=f_{P_{1}}\left(\left.u\right|_{P_{1}}\right)+f_{P_{2}}\left(\left.u\right|_{P_{2}}\right) .
$$

(ii) Continuity. For any set $A$ in the minimal field containing the collection of open subsets of the body, any sequence $P_{i}$ of sub-bodies converging to $A$ (in the sense that for every element $x$ in $A$ there is an integer $i_{0}$ so that $x \in P_{i}$ for every $i>i_{0}$ and every point that is contained in an infinite number of sets in the sequence is contained in $A$ ) and every $u \in C^{1}\left(h^{*} \tau_{M}\right)$, the sequence $f_{P_{i}}\left(\left.u\right|_{P_{i}}\right)$ converges and its limit is independent of the particular sequence of sub-bodies $P_{i}$. 
(iii) Boundedness. There is a finite bound $K$ such that for any sub-body $P$ and any $u \in C^{1}\left(h^{*} \tau_{M}\right),\left|f_{P}\left(\left.u\right|_{P}\right)\right|<K\left\|\left.u\right|_{P}\right\|$.

In that paper it was also shown that if a bounded set function on the collection of sub-bodies is given then additivity and continuity (in the sense mentioned above) are sufficient conditions so that the set functions may be extended to a measure on $B$ uniquely.

While these results are proved for the case where space is $\mathbb{R}^{n},{ }^{13}$ it may be shown ${ }^{11}$ that for a general differentiable manifold, the analytical question of the consistency of a force systems and a stress may be settled locally, in the domain of a chart. Hence, all these results apply to the general framework considered here.

We recall that the foregoing analysis considered the microforce $f_{2}$ so that the basic representation expression may be written specifically as

$$
f_{2}\left(u_{2}\right)=\int_{B} j\left(u_{2}\right) d \Sigma^{M},
$$

where the subscript $M$ denotes the fact that the stress represents the micro-force $f_{2}$. As we mentioned earlier, the representation theorem for the micro-force holds in particular for the geometric component of the force $f_{1}$ by putting $M=E$. Thus we may write

$$
f_{1}\left(u_{1}\right)=\int_{B} j\left(u_{1}\right) d \Sigma^{E},
$$

where the superscript $E$ indicates the stress representing the macro-force $f_{1}$. The representation of a force on a body with microstructure can be written therefore as

$$
f(u)=f_{1}\left(u_{1}\right)+f_{2}\left(u_{2}\right)=\int_{B} j\left(u_{1}\right) d \Sigma^{E}+\int_{B} j\left(u_{2}\right) d \Sigma^{M}
$$

Using the notation $j(u)$ for the pair $\left(j\left(u_{1}\right), j\left(u_{2}\right)\right), \boldsymbol{\Sigma}$ for the pair $\left(\Sigma^{E}, \Sigma^{M}\right)$ we may write the representation of forces in the form

$$
f(u)=\int_{B} j(u) d \mathbf{\Sigma} .
$$

\section{The Decomposition into a Self-Force and a Stress}

One would tend to refer to the components $\Sigma_{q}$ of the stress measures as the components of a vector measure called self-force and to the components $\Sigma_{P}^{I}$ as the components of a tensor valued measure called the microstress. However, the transformation rules for these entities imply that under a change of coordinates the transformed components $\Sigma_{q^{\prime}}$ depend not only on $\Sigma_{q}$ but also on $\Sigma_{q}^{I}$. We conclude therefore that at this level of generality the stress measure cannot be decomposed invariantly into a self-force and microstress. The geometric structure needed in order that such a decomposition can be defined invariantly is a connection on the manifold $M$. 
A connection on the manifold $M$ enables us to define invariantly the covariant derivative $\nabla u$ of a section $u: M \rightarrow T M$. The covariant derivative operator is a linear mapping $\nabla: C^{1}\left(\tau_{M}\right) \rightarrow C^{0}(L(T M, T M))$, where we use $C^{1}\left(\tau_{M}\right)$ to denote the collection of differentiable sections of $T M$ and we use $C^{0}(L(T M, T M))$ to denote the collection of continuous sections of the tensor bundle of linear mappings $T M \rightarrow T M$. The covariant derivative operator on $T M$ induces for a given $h \in$ $C^{\mathbf{1}}(B, M)$ a covariant derivative

$$
\nabla_{h}: C^{1}\left(h^{*} \tau_{M}\right) \rightarrow C^{0}\left(L\left(T B, h^{*} \tau_{M}\right)\right),
$$

and hence a connection on $h^{*} \tau_{M}$. The components of the Christoffel symbol for $h^{*} \tau_{M}$ are given by $\Gamma_{q I}^{p}=\Gamma_{q r}^{p} h_{, I}^{r}$, where $\Gamma_{q r}^{p}$ are the components of the Christoffel symbol for $T M$. The components of the covariant derivative of $u \in C^{1}\left(h^{*} \tau_{M}\right)$ are given therefore by

$$
u_{\mid I}^{p}=u_{, I}^{p}+\Gamma_{q r}^{p} u^{q} h_{, I}^{r}
$$

\section{Example: The Riemannian structure of the projective plane}

The fact that the tangent planes to $P^{2}$ may be embedded naturally in $V$, the tangent space of the Euclidean space, implies that we may use the inner product in $V$ in order to define inner products on the various tangent spaces. Thus, for a microstate $n$, two micro-velocities $v$ and $v^{\prime}$ belonging to $T_{n} P^{2}$, the inner product $v \cdot v^{\prime}$ is defined as the inner product of the corresponding angular velocities $\boldsymbol{\omega}$ and $\boldsymbol{\omega}^{\prime}$.

Consider an element $n$ of the projective plane and a Euclidean plane perpendicular to the line $n$ at a point $p$ on the line having a unit distance from $O$ (see Fig. 4). In addition, let $x^{i}$ be a set of Cartesian coordinates in the Euclidean space whose origin is at $O$ and such that the first two coordinate axes $x^{1}$ and $x^{2}$ are parallel to the perpendicular Euclidean plane. Thus, the coordinates $x^{1}$ and $x^{2}$ of the intersection of any line in $P^{2}$ with the plane serve as coordinates for a chart in a neighborhood of $n$. We will refer to this chart as a chart centered at $n$.

In order to calculate the components of the metric tensor at $n \in P^{2}$, let $v$ be an element of $T_{n} P^{2}$. As mentioned earlier we identify $v$ with the unique element $\boldsymbol{\omega}$ of $V$ representing it. By our construction, the components of $\overline{O p}$ are $(0,0,-1)$ and the components of $\omega$ (which is in the $x^{1}, x^{2}$ plane) are $\left(\omega^{1}, \omega^{2}, 0\right)$. It follows that the components of the velocity of point $p$ are $\left(-\omega^{2}, \omega^{1}, 0\right)$. By the construction of the chart centered at $p$, the representatives $\left(v^{1}, v^{2}\right)$ of the micro-velocity $v$ will be given by $\left(v^{1}, v^{2}\right)=\left(-\omega^{2}, \omega^{1}\right)$. In order to evaluate the components $g_{i j}$ of the metric tensor we use

$$
g_{i j} v^{i} v^{\prime j}=v \cdot v^{\prime}=\boldsymbol{\omega} \cdot \boldsymbol{\omega}^{\prime}
$$

that holds for every two micro-velocity vectors $v$ and $v^{\prime}$ and the angular velocities $\omega$ and $\omega^{\prime}$ representing them. Using the relation $\left(v^{1}, v^{2}\right)=\left(-\omega^{2}, \omega^{1}\right)$ we may 


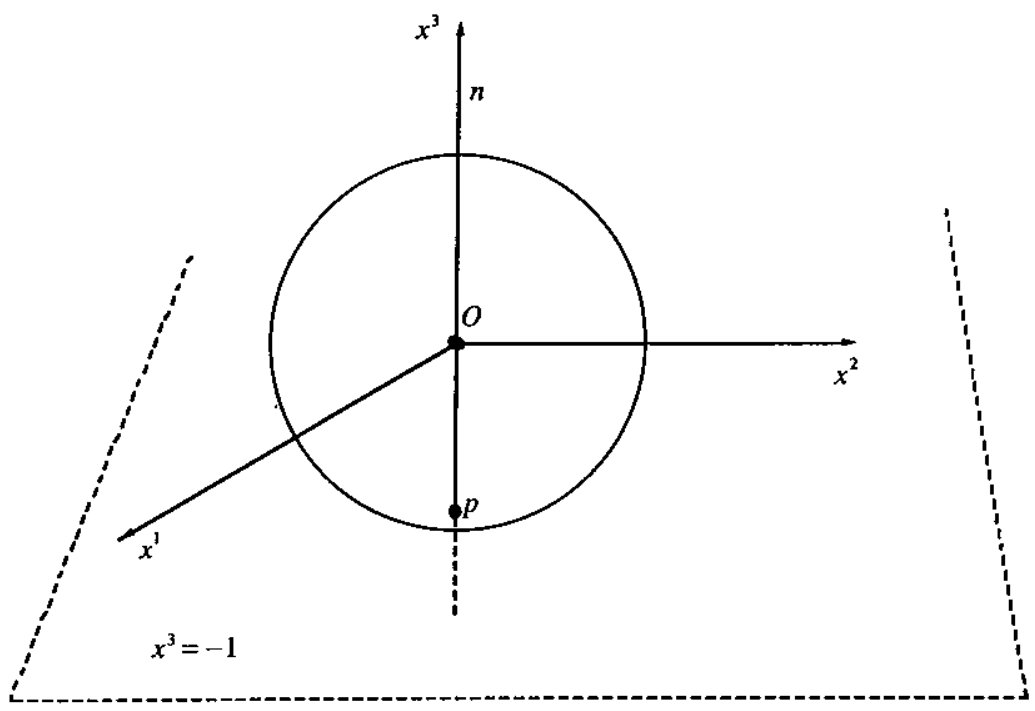

Fig. 4. A chart centered at $n$.

rewrite the last equation as

$$
g_{11}\left(-\omega^{2}\right)\left(-\omega^{\prime 2}\right)+2 g_{12}\left(-\omega^{2}\right) \omega^{1}+g_{22} \omega^{1} \omega^{1}=\omega^{1} \omega^{1}+\omega^{2} \omega^{2} .
$$

We conclude that in the chart centered at $n$, the metric tensor is given by $g_{\alpha \beta}=\delta_{\alpha \beta}$ (the Kronecker $\delta$ ).

Since every linear functional $f$ defined on an inner product space may be represented by a vector $u_{f}$ so that $f(v)=u_{f} \cdot v$ for any vector $v$, we conclude that forces may be identified with tangent vectors in the plane perpendicular to $n$. We also note that since the metric tensor is the identity tensor when we use a chart centered at $p$, the components of $f$ and the components of $u_{f}$ in such a chart are the same. Physically, these forces are the torques perpendicular to the axis of the molecule.

The covariant derivative of a vector field $w: P^{2} \rightarrow T P^{2}$ is a tensor field $\nabla w$ over $P^{2}$ whose components relative to a chart are denoted by $w_{\mid \beta}^{\alpha}$. The components of $\nabla w$ are given by $w_{\mid \beta}^{\alpha}=w_{, \beta}^{\alpha}+\Gamma_{\beta \gamma}^{\alpha} w^{\gamma}$. The Christoffel symbols $\Gamma_{\beta \gamma}^{\alpha}$ are induced by the metric structure on $P^{2}$ and they may be calculated by the relation

$$
\Gamma_{\alpha \beta}^{\gamma}=\frac{1}{2} g^{\gamma \delta}\left(g_{\beta \delta, \alpha}+g_{\alpha \delta, \beta}-g_{\alpha \beta, \delta}\right)
$$

Writing the relation between the components $\left(v^{1}, v^{2}\right)$ of a micro-velocity $v$ at an element $n^{\prime}$ in a neighborhood of $n$ and the components of the angular velocity representing it, we may find the expression for the metric tensor in a neighborhood of $n$. It turns out that the partial derivatives at $n$ of the components of the metric tensor in the chart centered at $n$ vanish, and it follows that the same holds for the Christoffel symbols. 
We conclude that the covariant derivative at $n$ is equal to the partial derivative in the chart centered at $n$. As a result, lines in the $\left(x^{1}, x^{2}\right)$ plane correspond infinitesimally to geodesics in $P^{2}$. In addition, since the inner product is identical to that of the sphere, the geodesics of $P^{2}$ correspond locally to geodesics on the sphere.

With the additional structure available, we can replace the jet extension mapping in the procedure for the representation of forces by stresses by the mapping

$$
\begin{gathered}
j_{\Gamma}: C^{1}\left(h^{*} \tau_{M}\right) \rightarrow C^{0}\left(h^{*} \tau_{M} \times_{B} L\left(T B, h^{*} \tau_{M}\right)\right) \\
j_{\Gamma}(u)=\left(u, \nabla_{h} u\right)
\end{gathered}
$$

Here, $h^{*} \tau_{M} \times_{B} L\left(T B, h^{*} \tau_{M}\right)$ is the vector bundle over $B$ whose fiber at $X$ is the Cartesian product $\left(h^{*} \tau_{M}\right)_{X} \times L\left(T_{X} B,\left(h^{*} \tau_{M}\right)_{X}\right)$. Similarly to $j$, the mapping $j_{\Gamma}$ is a linear injection which is a homeomorphism of $C^{1}\left(h^{*} \tau_{M}\right)$ onto its image. We may conclude that every force $f \in C^{1}\left(h^{*} \tau_{M}\right)^{*}$ is of the form $f=j_{\Gamma}^{*}(\Sigma)$ for some $\Sigma \in C^{0}\left(h^{*} \tau_{M} \times_{B} L\left(T B, h^{*} \tau_{M}\right)\right)^{*}$.

The dual space $C^{0}\left(h^{*} \tau_{M} \times_{B} L\left(T B, h^{*} \tau_{M}\right)\right)^{*}$ contains measures on $B$ valued in the dual bundle $\left(h^{*} \tau_{M} \times_{B} L\left(T B, h^{*} \tau_{M}\right)\right)^{*}=\left(h^{*} \tau_{M}\right)^{*} \times_{B} L\left(T B, h^{*} \tau_{M}\right)^{*}=$ $\left(h^{*} \tau_{M}\right)^{*} \times_{B} L\left(h^{*} \tau_{M}, T B\right)$. It follows that the measure $\Sigma$ can be decomposed invariantly into two measures: the first is valued in $\left(h^{*} \tau_{M}\right)^{*}$ and we will refer to it as the self-force, the second is valued in $L\left(h^{*} \tau_{M}, T B\right)$ and we will refer to it as the microstress measure.

Thus, for the case where $M$ is endowed with a connection the fact that the stress $\Sigma=\left(\Sigma_{0}, \Sigma_{1}\right)$ represents the force $f$, may be expressed in the form

$$
f(u)=\int_{B} d \Sigma_{0}(u)+\int_{B} d \Sigma_{1}\left(\nabla_{h} u\right) \text { for all } u .
$$

The actions $\int d \Sigma_{0}(u)$ and $\int d \Sigma_{1}\left(\nabla_{h} u\right)$ are expressed in local coordinates in the forms $\int u^{p} d \Sigma_{0 p}, \int u_{\mid I}^{p} d \Sigma_{1 p}^{I}$, respectively. While both $u_{\mid I}^{p}$ and the measures $\Sigma_{1 p}^{I}$ are local representatives that depend on the charts chosen, $u_{\mid I}^{p} \Sigma_{1 p}^{I}$ is a local expression which is invariant under changes of charts and it represents a real measure over the body $B$ which we will denote by $\operatorname{tr}\left(\nabla_{h} u \circ \Sigma_{1}\right)=\operatorname{tr}\left(\Sigma_{1} \circ \nabla_{h} u\right)$. We may write therefore

$$
f(u)=\int_{B} d \Sigma_{0}(u)+\int_{B} \operatorname{tr}\left(\nabla_{h} u \circ d \Sigma_{1}\right) .
$$

If we write specifically $f_{2}$ for the micro-force in the last equation and use $\Sigma_{0}^{M}$, $\Sigma_{1}^{M}$ to indicate that the self-force and microstress measure corresponding to the micro-force we have

$$
f_{2}\left(u_{2}\right)=\int_{B} d \Sigma_{0}^{M}\left(u_{2}\right)+\int_{B} \operatorname{tr}\left(\nabla_{h} u_{2} \circ d \Sigma_{1}^{M}\right) .
$$


The decomposition of the stress into the self-force and microstress measures holds in particular for the geometrical component of the force as the Euclidean space has the natural connection induced by the Euclidean structure. We will use $D u_{1}$ to denote the covariant derivative of the vector field $u_{1}$ and $\Sigma_{0}^{E}, \Sigma_{1}^{E}$ to denote the geometric self-force and stress measures. We may write therefore

$$
f_{1}\left(u_{1}\right)=\int_{B} d \Sigma_{0}^{E}\left(u_{1}\right)+\int_{B} \operatorname{tr}\left(D u_{1} \circ d \Sigma_{0}^{E}\right) .
$$

Thus, the expression for the representation of forces by stresses in the case where the manifold $M$ is equipped with a connection takes the form

$$
f(u)=\int_{B} d \Sigma_{0}^{E}\left(u_{1}\right)+\int_{B} \operatorname{tr}\left(D u_{1} \circ d \Sigma_{1}^{E}\right)+\int_{B} d \Sigma_{0}^{M}\left(u_{2}\right)+\int_{B} \operatorname{tr}\left(\nabla_{h} u_{2} \circ d \Sigma_{1}^{M}\right) .
$$

\section{Symmetry Considerations and the Associated Balance Laws}

The notions of a total force and equilibrium cannot be formulated in the general framework we had so far. The geometrical structure needed for the introduction of these notions is that of a Lie group on the manifold $S=E \times M$ containing the possible states of the various material points. In fact, we may consider the situation where a configuration of the body is a mapping $\kappa: B \rightarrow S$ for a general manifold $S$ and then specialize it to the case where $S=E \times M$. We will keep the notation $Q$ for the configuration space of $B$ in $S$ etc.

Assume that a Lie group $G$ acts on the manifold $S$ on the left. Thus, we have a mapping $\phi: G \times S \rightarrow S$ such that the restriction $\phi_{\mid g}:\{g\} \times S \rightarrow S$ is a diffeomorphism of $S$ for every $g \in G$, and at the identity $e$ of the Lie group, $\phi_{\mid e}=i d_{M}$. The action $\phi$ induces a left action

$$
\Phi: G \times Q \rightarrow Q
$$

on the configuration space $Q$ that is given by $\Phi(g, h)=\phi_{\mid g} \circ h$ for every $h \in Q$. The restriction, $\Phi_{\mid h}: G \times\{h\} \rightarrow Q$, will be denoted by $\Phi_{h}$. Its image is the collection of micro-configurations that may be obtained from $h$ by the action of elements of the group $G$.

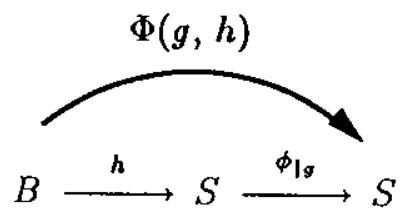

We consider the partial tangent mapping $T_{1} \phi: T G \times S \rightarrow T S$, and in particular, its restriction to the identity $\left(T_{1} \phi\right)_{\mid e}: T_{e} G \times S \rightarrow T S$. We denote by $i_{1}\left(T_{1} \phi\right)_{\mid e}$ : $T_{e} G \rightarrow C^{1}\left(\tau_{M}\right)$ the mapping such that for $\nu \in S, \xi \in T_{e} G, i_{1}\left(T_{1} \phi\right)_{\mid e}(\xi)(\nu)=$ 
$\left(T_{1} \phi\right)_{\mid e}(\xi, \nu)$. For each $\xi$ in the Lie algebra $T_{e} G, i_{1}\left(T_{1} \phi\right)_{\mid e}(\xi): S \rightarrow T S$ is a vector field on $S$. The vector field $i_{1}\left(T_{1} \phi\right)_{\mid e}(\xi)$ generates a vector field $u_{\xi}$ along $h$ defined by

$$
u_{\xi}=i_{1}\left(T_{1} \phi\right)_{\mid e}(\xi) \circ h=h^{*}\left(i_{1}\left(T_{1} \phi\right)_{\mid e}(\xi)\right) .
$$

It follows that, $u_{\xi}=h^{*}\left(i_{1}\left(T_{1} \phi\right)_{\mid e}(\xi)\right)$ is an element of the tangent space $T_{h} Q$. A simple calculation shows that

$$
u_{\xi}=T\left(\Phi_{h}\right)_{\mid e}(\xi),
$$

where the linear mapping $T\left(\Phi_{h}\right)_{\mid e}: T_{e} G \rightarrow T_{h} Q$ is the restriction to the Lie algebra of the derivative of the action of $G$ on the configuration space as restricted to $h$.

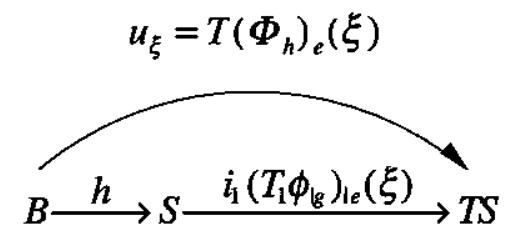

The dual mapping $T\left(\Phi_{h}\right)_{e}^{*}:\left(T_{h} Q\right)^{*} \rightarrow\left(T_{e} G\right)^{*}$ maps forces acting on the body at the configuration $h$ into elements of the dual of the Lie algebra. We will refer to elements of the dual of the Lie algebra as generalized resultants and to $r=$ $T\left(\Phi_{h}\right)_{j e}^{*}(f), f \in\left(T_{h} Q_{2}\right)^{*}$, as the generalized resultant of the force $f$. Thus, if $r$ is the generalized resultant of the force $f$, we have

$$
\begin{gathered}
r(\xi)=f\left(T\left(\Phi_{h}\right)_{\mid e}(\xi)\right)=f\left(i_{1}\left(T_{1} \phi\right)_{\mid e}(\xi) \circ h\right)=f\left(u_{\xi}\right) . \\
\left(T_{e} G\right)^{*} \stackrel{T\left(\Phi_{h}\right)_{\text {le }}^{*}}{\longleftarrow}\left(T_{h} Q\right)^{*} \\
T_{e} G \stackrel{T\left(\Phi_{h}\right)_{\text {le }}}{\longrightarrow} T_{h} Q \\
\left(T_{e} G\right)^{*} \stackrel{T\left(\Phi_{h}\right)_{\text {le }}^{*}}{\longleftarrow}\left(T_{h} Q\right)^{*}=C^{1}\left(h^{*} \tau_{S}\right)^{*} \stackrel{j^{*}}{\longleftarrow} C^{0}\left(J\left(h^{*} \tau_{S}\right)\right)^{*} \\
T_{e} G \stackrel{T\left(\Phi_{h}\right)_{\mid e}}{\longrightarrow} T_{h} Q=C^{1}\left(h^{*} \tau_{S}\right) \stackrel{j}{\longrightarrow} C^{0}\left(J\left(h^{*} \tau_{S}\right)\right)
\end{gathered}
$$

Given a stress $\boldsymbol{\Sigma}$, the forces $f_{P}$ induced by the stress $\boldsymbol{\Sigma}$ on the various sub-bodies give the generalized resultants

$$
r_{P}(\xi)=\int_{P} j\left(u_{\xi}\right) d \mathbf{\Sigma}
$$

on the various sub-bodies. We first note that $r_{P}$ may be regarded as the value of the set function $\rho$ defined on the collection of sub-bodies and valued in $\left(T_{e} G\right)^{*}$ such that $\rho(P)=r_{P}$. The set function $\rho$ has the following properties. 
(i) For two disjoint sub-bodies $P_{1}, P_{2}$

$$
\begin{aligned}
\rho\left(P_{1} \cup P_{2}\right)(\xi) & =\int_{P_{1}} j\left(u_{\xi}\right) d \mathbf{\Sigma}+\int_{P_{2}} j\left(u_{\xi}\right) d \mathbf{\Sigma} \\
& =\left(\rho\left(P_{1}\right)+\rho\left(P_{2}\right)\right)(\xi)
\end{aligned}
$$

so that $\rho$ is additive.

(ii) If $A$ is a set in the minimal field of subsets of $B$ containing the open subsets and $P_{i} \rightarrow A$, then

$$
\rho\left(P_{i}\right)(\xi)=\int_{P_{i}} j\left(u_{\xi}\right) d \mathbf{\Sigma} \rightarrow \int_{A} j\left(u_{\xi}\right) d \mathbf{\Sigma}
$$

which is independent of the particular sequence $P_{i}$ by the properties of $\boldsymbol{\Sigma}$ as a measure.

It follows from the results cited earlier ${ }^{13}$ that the set function $\rho$ may be extended to a $\left(T_{e} G\right)^{*}$ valued Borel measure on $B$ for which we will also use the notation $\rho$. Using the notation introduced above we may write

$$
\rho(P)(\xi)=\int_{P} d \mathbf{\Sigma}\left(\left(j \circ T\left(\Phi_{h}\right)_{\mid e}\right)(\xi)\right),
$$

and it follows that the measure $\rho$ is given by

$$
\rho(P)=\Sigma_{\mid P} \circ j \circ T\left(\Phi_{h}\right)_{\mid e} .
$$

The measure $\rho$ may be described as the distribution of the generalized resultant in the body as induced by the stress $\boldsymbol{\Sigma}$.

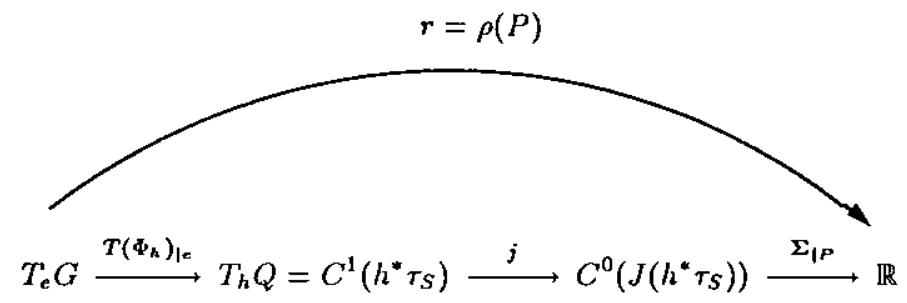

We will say that a force $f$ at the configuration $h$ is $G$-invariant if the generalized resultant associated with it vanishes. Thus, for a $G$-invariant force $f$ the virtual power $f\left(T\left(\Phi_{h}\right)_{\mid e}(\xi)\right)=f\left(u_{\xi}\right)=0$ for each $\xi$ in the Lie algebra $T_{e} G$, i.e. the virtual power performed by the force vanishes for every micro-virtual-displacement induced by a symmetric vector field. The equation $T\left(\Phi_{h}\right)_{\mid}^{*}(f)=0$, representing the condition that $f$ is $G$-invariant is the balance law associated with the group $G$. 
The restriction that the balance law imposes on the stress measure $\Sigma$ follows from the representation of forces by stresses

$$
f\left(u_{\xi}\right)=\int_{B} j\left(u_{\xi}\right) d \mathbf{\Sigma}=0
$$

for all $\xi \in T_{e} G$, so that we may write the balance law as

$$
\boldsymbol{\Sigma} \circ j \circ T\left(\Phi_{h}\right)_{\mid e}=\left(T\left(\Phi_{h}\right)_{\mid e}\right)^{*}\left(j^{*}(\boldsymbol{\Sigma})\right)=0 \in\left(T_{e} G\right)^{*}
$$

where $\left(T\left(\Phi_{h}\right)_{\mid e}\right)^{*}$ is the adjoint of the linear mapping $T\left(\Phi_{h}\right)_{\mid e}$.

We will say that the stress $\Sigma$ is $G$-invariant if all the forces $\left\{f_{P}\right\}$ it induces on the various sub-bodies are $G$-invariant. Hence, for a $G$-invariant stress the resultants $\rho(P)$ vanish for each sub-body $P$. It follows that the measure $\rho$ vanishes and the condition for $\boldsymbol{\Sigma}$ is

$$
\boldsymbol{\Sigma} \circ j \circ h^{*} \circ i_{1}\left(T_{1} \phi\right)_{\mid e}=0 .
$$

This equation is a generalization of the conditions that the self-force and the antisymmetric part of the stress field vanish in continuum mechanics. It generalizes these conditions in two aspects. It applies to the general case of a differentiable manifold acted upon by a lie group and it holds for stresses as irregular as Borel measures.

\section{Example: The balance laws for liquid crystals}

In this example, in order to simplify the notation, we will fix an origin $x_{0} \in E$ so that we may identify $E$ with its tangent space $V$. It will be evident eventually that the results are independent of $x_{0}$. Thus, for liquid crystals, $S=V \times P^{2}$ and we use the action of the proper Euclidean group $G^{E}=V \times \mathrm{SO}(3)$ on $V$ in order to induce an action of $G^{E}$ on $S$. Here, $\mathrm{SO}(3)$ denotes the proper orthogonal group.

We will denote by $\phi^{E}: G^{E} \times V \rightarrow V$ the action of the Euclidean group on $V$. For $g=(\mathbf{w}, O) \in G^{E}, \phi(g, \mathbf{v})=\mathbf{w}+O(\mathbf{v})$. For the Lie algebra we have $T_{e} G^{E}=V \times \mathrm{so}(3)$, where so(3) is the vector space of skew symmetric linear transformations of $V$. For an element $\xi=(\eta, \zeta) \in T_{e} G^{E}$, we have $T_{1} \phi_{\mid \mathrm{e}}^{E}(\xi, \mathbf{w})=\eta+\zeta(\mathbf{w})$. We will identify an element of $\zeta \in \operatorname{so}(3)$ with the axial vector $\zeta$ representing it so that $T_{1} \phi_{\mid e}^{E}(\xi, w)=\eta+\zeta \times \mathbf{w}$.

The distribution $\rho^{E}$ of the resultant geometric force $f_{1}$ represented by the self-force $\Sigma_{0}^{E}$ and stress $\Sigma_{1}^{E}$ is given by

$$
\rho^{E}(P)(\xi)=\int_{P} d \Sigma_{0}^{E}\left(\eta+\zeta \circ h_{1}\right)+\int_{P} d \Sigma_{1}^{E}\left(D\left(\zeta \circ h_{1}\right)\right)
$$

where we note that $\zeta \circ h_{1}(X)=\zeta \times h_{1}(X)$. Since $\zeta$ is constant over the body, we may write $\Sigma_{1}^{E}\left(D\left(\zeta \circ h_{1}\right)\right)=\Sigma_{1}^{E}\left(\zeta \circ D h_{1}\right)=\zeta \circ\left(T h_{2} \circ \Sigma_{1}^{E}\right)$. We define the 
Cauchy stress (geometric stress) $s^{E}$ by $s^{E}=T h_{1} \circ \Sigma_{1}^{E}$ and the distribution of the geometric resultant force may be expressed as

$$
\begin{aligned}
\rho^{E}(P)(\xi) & =\int_{P} d \Sigma_{0}^{E}\left(\boldsymbol{\eta}+\zeta \times h_{1}\right)+\zeta \circ \int_{P} d s^{E} \\
& =\int_{P} d \Sigma_{0}^{E}\left(\boldsymbol{\eta}+\zeta \times h_{1}\right)+2 \zeta \cdot \int_{P} d\left[s^{E}\right],
\end{aligned}
$$

where $\left[s^{E}\right]$ is the axial vector valued measured corresponding to the skew symmetric part of $s^{E}$

The action of the proper Euclidean group on the tangent space to the Euclidean space induces an action of the rotational group $\mathrm{SO}(3)$ on the projective plane. Assume that an element $g=(\mathbf{w}, O) \in G^{E}$ is given. For any line $n$ we may choose a vector $\mathbf{v}$ such that $n=\operatorname{pr}(\mathbf{v})$ and define $\phi^{M}(O, n)=\operatorname{pr}(O(\mathbf{v}))$, where $\mathrm{pr}$ is the projection of the tangent of the Euclidean space into $P^{2} \mathrm{de}-$ fined above. The mapping $\phi^{M}: \operatorname{SO}(3) \times P^{2} \rightarrow P^{2}$ is a smooth action of the rotation group on the collection of lines. Since the inner product on $P^{2}$ was induced by the inner product in the Euclidean space, it is clear that $\phi_{\mid O}^{M}$ is an isometry of $P^{2}$ for any rotation $O$. The partial tangent of $\phi^{M}$ at the identity $T_{1} \phi_{\mid e}^{M}: T_{e} \mathrm{SO}(3) \times P^{2} \rightarrow T P^{2}$ will generate velocity fields on the projective plane out of infinitesimal rotations of the Euclidean space. Clearly, for a given line $n$ in the projective plane and an infinitesimal rotation $\zeta \in \operatorname{so}(3)$, the micro-velocity $T_{1} \phi^{M}(\zeta, n)$ is represented by an angular velocity $\omega$ which is the component of the infinitesimal rotation vector perpendicular to the line $n$. We will use the notation $a$ : $\operatorname{so}(3) \rightarrow C^{1}\left(T P^{2}\right)$ for the mapping $i_{1}\left(T_{1} \phi\right)_{\mid e}$ defined earlier. We will also denote by $b: \operatorname{so}(3) \rightarrow C^{0}\left(L\left(T P^{2}, T P^{2}\right)\right)$ the mapping such that $\zeta \mapsto \nabla(a(\zeta))$. Finally, we may define the linear mapping $c: \operatorname{so}(3) \rightarrow C^{1}\left(T P^{2}\right) \times C^{0}\left(L\left(T P^{2}, T P^{2}\right)\right)$ by $c(\zeta)=(a(\zeta), b(\zeta))$.

For a given element $\zeta \in \operatorname{so}(3), a(\zeta)(n)$ is represented by the angular velocity field $\boldsymbol{\omega}_{\zeta}$ on $P^{2}$ which is given by $\boldsymbol{\omega}_{\zeta}(\mathbf{n})=\zeta-(\mathbf{n} \otimes \mathbf{n}) \zeta$, where $\mathbf{n}$ is a unit vector (in $V$ ) along the line $n$. Alternatively, using a chart centered at a line $n_{0}$, the components of $a(\zeta)(n)$ in this chart are $\left(u^{1}, u^{2}\right)=\left(-\zeta^{2}-x^{2} \zeta^{3}, \zeta^{1}+x^{1} \zeta^{3}\right)$, where the $x^{\alpha}$ are the coordinates of the generic point $n$ in the chart centered at $n_{0}$. (These are the components of the velocity of the point of intersection of the line $n$, rotating with angular velocity $\zeta$, with the plane $x^{1}, x^{2}$.) Using this chart the local representative of $a(\zeta)(n)$ is

$$
\left(\begin{array}{rrr}
0 & -1 & x_{2} \\
1 & 0 & x_{1}
\end{array}\right)\left(\begin{array}{l}
\zeta^{1} \\
\zeta^{2} \\
\zeta^{3}
\end{array}\right)
$$

and we will denote the components of the first matrix by $a_{i}^{\alpha}(n)$. Since the components of the covariant derivative $\nabla(a(\zeta))=b(\zeta)$ are identical to the partial 
derivatives for a chart centered at $n$, the components of the covariant derivative $b(\zeta)$ are given by

$$
u_{\mid 1}^{1}=0, \quad u_{\mid 2}^{1}=-\zeta^{3}, \quad u_{\mid 1}^{2}=\zeta^{3}, \quad u_{\mid 2}^{2}=0
$$

These may be written as $b(\zeta)_{\mid \beta}^{\alpha}=b_{\beta i}^{\alpha} \zeta^{i}$, where

$$
b_{\beta 3}^{\alpha}=\left(\begin{array}{rr}
0 & -1 \\
1 & 0
\end{array}\right)
$$

and the rest of the components vanish.

As the action of the elements of the Lie algebra on the liquid crystal microstructure is associated with angular velocities only, it is natural to refer to the elements of the dual of the Lie algebra as the moments of the micro-force. Thus, for a given force $f_{2}$ the resulting moments or total moment of the microforce over the body is given by $r^{M}(\xi)=f_{2}\left(a(\zeta) \circ h_{2}\right)$. Assume that a self-force $\Sigma_{0}^{M}$ and a microstress measure $\Sigma_{1}^{M}$ are given on $B$. Then, the resultant of the force induced on a sub-body $P$ is given by

$$
\rho^{M}(P)(\xi)=\int_{P} d \Sigma_{0}^{M}\left(a(\zeta) \circ h_{2}\right)+\int_{P} \operatorname{tr}\left(\nabla_{h_{2}}\left(a(\zeta) \circ h_{2}\right) \circ d \Sigma_{1}^{M}\right)
$$

Note that $\nabla_{X}\left(a(\xi) \circ h_{2}\right)(X)=\nabla a(\xi)\left(h_{2}(X)\right) \circ T h_{2}(X)$, and we may write this as $\nabla_{X}\left(a(\xi) \circ h_{2}\right)=\left(\nabla a(\xi) \circ h_{2}\right) \circ T h_{2}$. It follows that the expression for the resultant of the micro-force may be rewritten as

$$
\rho^{M}(P)(\xi)=\int_{P} d \Sigma_{0}^{M}\left(a(\zeta) \circ h_{2}\right)+\int_{P} \operatorname{tr}\left(\left(\nabla a(\zeta) \circ h_{2}\right) \circ T h_{2} \circ d \Sigma_{1}^{M}\right)
$$

We now define the Cauchy microstress measure by $s^{M}=T h_{2} \circ \Sigma_{1}^{M}$ and using the notation introduced above we obtain

$$
\rho^{M}(P)(\xi)=\int_{P} d \Sigma_{0}^{M}\left(a(\zeta) \circ h_{2}\right)+\int_{P} \operatorname{tr}\left(\left(b(\zeta) \circ h_{2}\right) \circ d s^{M}\right)
$$

Assuming that the image of $P$ is contained in a chart centered at a line $n_{0}$ and using the local expressions for $a$ and $b$ we can write $r_{P}^{M}(\xi)$ in the form

$$
\rho^{M}(P)(\xi)=\zeta^{i}\left[\int_{P} a_{i}^{\alpha}\left(h_{2}(X)\right) d \Sigma_{0 \alpha}^{M}(X)+\int_{P} b_{\beta i}^{\alpha}\left(h_{2}(X)\right) d s_{\beta}^{M \alpha}(X)\right] .
$$

It follows that the local expression for the mapping $\left(T\left(\Phi_{h}^{M}\right)_{\mid e}\right)^{*} \circ j^{*}$ giving the total moment of the stress is given by

$$
\int_{P} a_{i}^{\alpha}\left(h_{2}(X)\right) d \Sigma_{0 \alpha}^{M}(X)+\int_{P} b_{\beta i}^{\alpha}\left(h_{2}(X)\right) d s_{\beta}^{M \alpha}(X) .
$$


We now assume that the stress $\Sigma=\left(\left(\Sigma_{0}^{E}, \Sigma_{1}^{E}\right),\left(\Sigma_{0}^{M}, \Sigma_{1}^{M}\right)\right)$ is $G$-invariant. We have

$$
\begin{aligned}
\rho^{E}(P)(\xi)= & \int_{P} d \Sigma_{0}^{E}\left(\boldsymbol{\eta}+\zeta \times h_{1}\right)+2 \zeta \cdot \int_{P} d\left[s^{E}\right]+\int_{P} d \Sigma_{0}^{M}\left(a(\zeta) \circ h_{2}\right) \\
& +\int_{P} \operatorname{tr}\left(\left(b(\zeta) \circ h_{2}\right) \circ d s^{M}\right) \\
= & 0 .
\end{aligned}
$$

Since we may choose $\zeta=0$ and an arbitrary vector $\boldsymbol{\eta}$, we conclude immediately that $\Sigma_{0}^{E}(P)=0$. Since this holds for any sub-body $P$ it follows ${ }^{13}$ that $\Sigma_{0}^{E}=0$. This result clearly corresponds to the balance of forces in mechanics. We consider now a sub-body $P$ that is contained in a chart centered at some line $n_{0}$. The expression for the resultant becomes

$$
2 \zeta^{i} \cdot \int_{P} d\left[s^{E}\right]_{i}+\int_{P} a_{i}^{\alpha}\left(h_{2}(X)\right) d \Sigma_{0 \alpha}^{M}(X)+\int_{P} b_{\beta i}^{\alpha}\left(h_{2}(X)\right) d s_{\beta}^{M \alpha}(X)=0
$$

Substituting $\zeta^{i}=\delta^{1 i}$ we obtain

$$
2\left[s^{E}\right]_{1}(P)+\int_{P} a_{1}^{\alpha} d \Sigma_{0 \alpha}^{M}+\int_{P} b_{\beta 1}^{\alpha} d s_{\beta}^{M \alpha}=0
$$

and substituting the values of $a_{1}^{\alpha}$ and $b_{\beta 1}^{\alpha}$ we obtain

$$
2\left[s^{E}\right]_{1}(P)+\Sigma_{02}^{M}(P)=0,
$$

and it follows that $\left[s^{E}\right]_{1}+\Sigma_{02}^{M}=0$. Similarly, for $\zeta^{i}=\delta^{2 i}$, the balance law is

$$
2\left[s^{E}\right]_{2}(P)-\Sigma_{01}^{M}(P)=0
$$

and we have $\left[s^{E}\right]_{2}-\Sigma_{01}^{M}=0$.

For $\zeta^{i}=\delta^{3 i}$, one obtains

$$
2\left[s^{E}\right]_{3}(P)+\int_{P}\left(x_{2} d \Sigma_{01}^{M}+x_{1} d \Sigma_{02}^{M}\right)+\left(s_{1}^{M 2}-s_{2}^{M 1}\right)(P)=0 .
$$

The treatment of this balance law is more involved because of the integral in the middle term. For short we will use the notation $A=2\left[s^{E}\right]_{3}+\left(s_{1}^{M 2}-s_{2}^{M 1}\right)$ and also $\mu=\left|\Sigma_{01}^{M}\right|+\left|\Sigma_{02}^{M}\right|$. From the balance law it is clear that the measure $A$ is absolutely continuous with respect to the measure $\mu$. Let $\hat{A}$ be the RadonNikodym derivative of $A$ with respect to $\mu$. We have

$$
|A(P)|=\left|\int_{P} \hat{A} d \mu\right|=\left|\int_{P}\left(x_{2} d \Sigma_{01}^{M}+x_{1} d \Sigma_{02}^{M}\right)\right| \leq r \int_{P} d \mu,
$$


where $r=\sup _{n \in P}\left\{\left|x_{1}\right|,\left|x_{2}\right|\right\}$ is the radius of the image of the set $P$ under the chart. We conclude that $\lim _{x_{\alpha} \rightarrow 0} \hat{A}=0$. Hence, denoting the densities of the various measures with respect to $\mu$ by superimposed carets we have

$$
2\left[\hat{s}^{E}\right]_{3}(0,0)+\left(\hat{s}_{1}^{M 2}-\hat{s}_{2}^{M 1}\right)(0,0)=0,
$$

and using $\left[s^{M}\right]$ for $s_{1}^{M 2}-s_{2}^{M 1}$ we finally obtain that

$$
\left[\hat{s}^{E}\right]_{3}+\left[\hat{s}^{M}\right]=0
$$

at any point when we use the chart centered at that point. If we assume that the measures $\Sigma_{1}^{M}, \Sigma_{2}^{E}$ may be represented by smooth densities with respect to the volume measure in the body, the last equation holds for these smooth axial vector fields.

The last three balance laws are clearly the balance of moments or moment of momentum.

It should be noted that by its definition, the Cauchy microstress measure and its symmetry properties do not represent completely the analogous properties of the Piola-Kirchhoff stress measures in cases where $T h_{2}$ is not of full rank. This is in contrast to the situation pertaining to $\left[s^{E}\right]$ where the axiom of material impenetrability implies that $T h_{1}$ is always of full rank. The simplest analog to the situation is the example of a collection of forces acting on a body and in which it happens that all forces are acting at the same point. The balance of moments will not provide information in addition to the balance of forces.

\section{Stresses Represented by Smooth Densities}

A particular case of the theory described above is the situation where the stress measure for either the macro-force or the micro-force may be given in terms of a smooth density with respect to the volume measure $V$ on $B$. As done previously we will consider first the microstress and later we shall specialize the results for the macrostress. In this context it is assumed that there is a smooth section $\sigma^{M}$ of the dual of the jet bundle $J\left(h^{*} \tau_{M}\right)^{*}$ such that $\Sigma^{M}=\sigma^{M} V$, hence, the representation of forces by stresses is of the form

$$
f_{2}\left(u_{2}\right)=\int_{B} \sigma^{M}(X)\left(j\left(u_{2}\right)(X)\right) d V, \quad \text { for all } u_{2} \in C^{1}\left(h^{*} \tau_{M}\right)=T_{h} Q_{2} .
$$

Clearly, the density $\sigma^{M}$ is represented locally in the form $\left(\sigma_{q}^{M}(X), \sigma_{p}^{M I}(X)\right)$. We will refer to the density $\sigma^{M}$ as stress field.

In the case where a connection is specified on the manifold $M$, the assumption that the stress may be represented by smooth densities implies that there are two smooth sections $\sigma_{0}^{M}, \sigma_{1}^{M}$ of the bundles $\left(h^{*} \tau_{M}\right)^{*}, L\left(h^{*} \tau_{M}, T B\right)$, respectively, such that the representation of forces by stresses is of the form

$$
f_{2}\left(u_{2}\right)=\int_{B} \sigma_{0}^{M}(X)\left(u_{2}(X)\right) d V+\int_{B} \sigma_{1}^{M}(X)\left(\nabla_{h_{2}} u_{2}(X)\right) d V
$$


The sections $\sigma_{0}^{M}, \sigma_{1}^{M}$ are of the forms $\sigma_{0 p}^{M}, \sigma_{1 p}^{M I}$, respectively. We will refer to $\sigma_{0}^{M}$, $\sigma_{1}^{M}$ as the micro self-force field and microstress field, respectively.

A standard calculation shows that

$$
\sigma_{1}^{M}(X)\left(\nabla_{h_{2}} u_{2}(X)\right)=\operatorname{div}\left(\sigma_{1}^{M}\left(u_{2}\right)\right)(X)-\left(\operatorname{div} \sigma_{1}^{M}\right)\left(u_{2}(X)\right),
$$

where the two divergence operators have different meanings: $\sigma_{1}^{M}(u)$ is a vector field $B \rightarrow T B$ and since $B$ is a submanifold of $\mathbb{R}^{3}$ the divergence is that of a vector field in $\mathbb{R}^{3}$, the divergence of $\sigma_{1}^{M}$ is the divergence of a tensor field valued in $L\left(h^{*} \tau_{M}, T B\right)$ using the connection given in $M$. Using the divergence theorem for $\operatorname{div}\left(\sigma_{1}^{M}\left(u_{2}\right)\right)$, we obtain

$$
f_{2}\left(u_{2}\right)=\int_{B}\left(\sigma_{0}^{M}-\operatorname{div} \sigma_{1}^{M}\right)\left(u_{2}\right) d V+\int_{B}\left(\sigma_{1}^{M}\left(u_{2}\right)\right) \cdot n d A .
$$

Using the definitions

$$
\begin{aligned}
& b_{M}=\sigma_{0}^{M}-\operatorname{div} \sigma_{1}^{M}: B \rightarrow\left(h^{*} \tau_{M}\right)^{*}, \\
& t_{M}=\left(\sigma_{1}^{M}\right)^{\mathrm{T}}(n): \partial B \rightarrow\left(h^{*} \tau_{M}\right)^{*},
\end{aligned}
$$

we have shown that under the assumptions made, the micro-force may be represented by differential forms $b_{M}, t_{M}$ as above in the form

$$
f_{2}\left(u_{2}\right)=\int_{B} b_{M}\left(u_{2}\right) d V+\int_{\partial B} t_{M}\left(u_{2}\right) d A .
$$

Clearly, the forms $b_{M}, t_{M}$ represent the micro-force $f_{2}$ uniquely. The covector field $b_{M}$ will be referred to as the micro body force field and the covector field $t_{M}$ will be referred to as the micro surface force field. In the case where the force $f_{2}$ is given in terms of a micro body force field $b_{M}$ and a micro surface force field $t_{M}$, the force is represented by the self-force field $\sigma_{0}^{M}$ and a microstress field $\sigma_{1}^{M}$ if the "equilibrium equation" and boundary conditions

$$
\begin{gathered}
\operatorname{div} \sigma_{1}^{M}+b_{M}=\sigma_{0}^{M} \quad \text { in } B \\
\left(\sigma_{1}^{M}\right)^{\mathrm{T}}(n)=t_{M} \quad \text { on } \quad \partial B
\end{gathered}
$$

are satisfied.

The analogous results for the macro-force are clearly

$$
\begin{gathered}
\operatorname{div} \sigma_{1}^{E}+b_{E}=\sigma_{0}^{E}, \quad \text { in } \quad B \\
\left(\sigma_{1}^{E}\right)^{\mathrm{T}}(n)=t_{E}, \quad \text { on } \quad \partial B
\end{gathered}
$$

where now the various fields are the classical fields of continuum mechanics and $\sigma_{0}^{E}$ is the macro self-force field that vanishes usually (as in the example of liquid crystals) as a result of symmetry requirements. 


\section{Latent Microstructure}

The term latent microstructure was introduced by Capriz ${ }^{1}$ for the case where the micro-configuration and micro-velocity of the body is determined by the geometric configuration and geometric velocity. Here, we only consider statics and do not have a notion of an "actual" velocity (in contrast to a generalized velocity or a virtual displacement), the most gereral situation is that the micro-configuration depends on the geometric configuration. In other words, we can consider holonomic latent microstructure only. Thus, it is assumed for the statics of material with latent microstructure, that there is a differentiable mapping

$$
\Lambda: Q_{1} \rightarrow Q_{2}
$$

that assigns to each geometrical configuration the corresponding microconfiguration. A curve in $Q_{1}$ will determine through $\Lambda$ a unique curve in $Q_{2}$ and any geometrical virtual displacement $u_{1}$ which is a tangent vector to $Q_{1}$ will determine the corresponding micro-virtual displacement $u_{2}$ by

$$
u_{2}=T \Lambda\left(u_{1}\right), \quad T \Lambda: T Q_{1} \rightarrow T Q_{2} .
$$

Thus, for any geometrical configuration $\kappa_{1} \in Q_{1}$ any micro-force $f_{2} \in\left(T_{\Lambda\left(\kappa_{1}\right)} Q_{2}\right)^{*}$ induces a geometrical force $f_{1}$ by

$$
f_{1}=T \Lambda^{*}\left(f_{2}\right) .
$$

Thus, the virtual power will be of the form

$$
\begin{aligned}
f(u) & =f_{1}\left(u_{1}\right)+f_{2}\left(u_{2}\right) \\
& =f_{1}\left(u_{1}\right)+f_{2}\left(T \Lambda\left(u_{1}\right)\right) \\
& =f_{1}\left(u_{1}\right)+T \Lambda^{*}\left(f_{2}\right)\left(u_{1}\right) \\
& =\left(f_{1}+T \Lambda^{*}\left(f_{2}\right)\right)\left(u_{1}\right) .
\end{aligned}
$$

It follows that the statics of a body with latent microstructure may be replaced by the statics of a simple body in $E$ if any geometrical force $f_{1}$ is replaced by $f_{1}+T \Lambda^{*}\left(f_{2}\right)$.

The description given here is more general than the theory of bodies with latent microstructure given in the works of Capriz ${ }^{1,2}$ in the sense that the latent microstructure may be nonlocal. This means that the microstate of a material point $X$ may depend on the geometrical states of material points other than $X$. The latent microstructure is local if the microstate of any material point $X$ depends on the geometrical state of $X$ only.

The question of the locality of the latent microstructure can be discussed in the framework as suggested by the author. ${ }^{12}$ In the general case where the latent 
microstructure is not necessarily local, the map $\Lambda$ depends on the sub-body under consideration. While so far we discussed configurations of the whole body $B$ only, we will indicate in the rest of this section the sub-body under consideration with an appropriate subscript. For example, our assumption that the body has a latent microstructure may be rewritten in the form

$$
\Lambda_{B}: Q_{B_{1}} \rightarrow Q_{B_{2}}
$$

This assumption means that the micro-configuration of $B$ is determined by the geometrical configuration of $B$ (and not a larger body). It is natural to extend this and require that the micro-configuration of each sub-body of $B$ will be determined by the geometrical configuration of that sub-body. Thus we make the following assumption.

Microstructure body determinism: The micro-configuration of each sub-body $P$ is determined by a mapping $\Lambda_{P}: Q_{P_{1}} \rightarrow Q_{P_{2}}$.

This assumption will be meaningless if for a point $X$ that belongs to two sub-bodies, the microstates of $X$ as determined by the latent microstructure for the two different sub-bodies will be contradicting. To make the previous assumption meaningful we have to assume:

Consistency: For any sub-body $P$ of $B$ and a material point $X$ we have

$$
\Lambda_{P}\left(\kappa_{1 \mid p}\right)(X)=\Lambda_{B}\left(\kappa_{1}\right)(X) .
$$

It follows from these two assumptions that the microstate of a point $X$ depends on the configuration at an arbitrary sub-body or an arbitrary open subset of $B$ containing $X$. These assumptions by themselves are not sufficient in order to guarantee that the microstate of $X$ depends on the value of the geometrical configuration and its derivatives at $X$.

We recall that $Q_{2}=C^{1}(B, M)$ and that $J\left(C^{1}(B, M)\right)$ is the jet bundle over $B$ whose fiber at any $X$ contains the possible values of tangents at $X$ of mappings in $C^{1}(B, M)$. Locally, elements of $J\left(C^{1}(B, M)\right)$ are represented by coordinates of the form $\left(\nu^{p}, \nu_{, I}^{r}\right)$. The jet extension mapping $j: C^{1}(B, M) \rightarrow C^{0}\left(J\left(C^{1}(B, M)\right)\right)$ is the continuous mapping that assigns to any micro-configuration $\kappa_{2}$ the continuous section of $J\left(C^{1}(B, M)\right)$ whose value at $X$ is the tangent of $\kappa_{2}$ at the material point $X$ (represented by $\left.\left(\nu^{p}(X), \nu_{, I}^{r}(X)\right)\right)$.

Earlier we made the assumption that $\Lambda$ is differentiable. Therefore the mapping

$$
j \circ \Lambda: Q_{1} \rightarrow C^{0}\left(J\left(C^{1}(B, M)\right)\right)
$$

is a continuous mapping. In addition, $j \circ \Lambda$ satisfies the assumptions of microstructure body determinism and consistency above. In the work concerning constitutive theory, ${ }^{12}$ mappings such as $j \circ \Lambda$ from the geometrical configuration space to a space 
of continuous functions on $B$ that are continuous and that satisfy these assumptions are considered in the context of the locality of constitutive relations. Using the results cited, ${ }^{12}$ it follows that the value of $\kappa_{2}$ and its tangent at $X$ depends continuously on the value of $\kappa_{1}$ and its tangent at $X$. In addition, the mapping that gives the value of $\kappa_{2}$ and its tangent at $X$ as a function of the corresponding values of $\kappa_{1}$, depends continuously on the material point $X$.

\section{Higher Order Theories}

Higher order theories are theories where stresses are tensors of orders higher than 2. These are used in order to model some physical phenomena that the traditional theories cannot describe. In a previous work, ${ }^{11}$ it was shown that such theories fit into the framework presented above very naturally. If we assume that configurations are $C^{n}$ mappings with $n>1$, rather than the case $n=1$ considered here, and we use the $C^{n}$ topology, we obtain by the same procedure described above the analogous higher order theory. ${ }^{11}$

In addition, the assumption that configurations are $C^{n}$ mappings and using the $C^{n}$ topology implies also for the case of bodies with latent microstructure that satisfy the assumptions of microstructure body determinism and consistency above that the following hold: The value of $\kappa_{2}$ and its first $n$ tangents (derivatives) at $X$ depends continuously on the value of $\kappa_{1}$ and its first $n$ tangents at $X$. In addition, the mapping that gives the value of $\kappa_{2}$ and its tangents at $X$ as a function of the corresponding values of $\kappa_{1}$ depends continuously on the material point $X$.

Thus, higher order theories extend naturally from the theory presented above.

\section{Acknowledgment}

This work was partially supported by the Paul Ivanier Center for Robotics Research and Production Management and it is the result of the discussions with G. Capriz and E. Virga during the author's visit to the University of Pisa kindly supported by the Italian N.R.C.

\section{References}

1. G. Capriz, Continua with latent microstructure, Arch. Rational Mech. Anal. 90 (1985) 43-56.

2. G. Capriz, Continua with Microstructure (Springer, 1989).

3. G. Capriz and E. Virga, Interactions of general continua with microstructure, Arch. Rational Mech. Anal. 109 (1990) 323-342.

4. I. E. Eliasson, Geometry of manifolds of maps, J. Differential Geom. 1 (1967) 169.

5. M. Epstein and R. Segev, Differentiable manifolds and the principle of virtual work in continuum mechanics, J. Math. Phys. 21 (1980) 1243-1245.

6. M. W. Hirsch, Differential Topology (Springer, 1976).

7. N. D. Mermin, The topological theory of defects in ordered media, Rev. Modern Phys. 51 (1979) 591-646.

8. W. Noll, Lectures on the foundations of continuum mechanics and thermodynamics, Arch. Rational Mech. Anal. 52 (1973) 62-92. 
9. R. Palais, Foundations of Global Nonlinear Analysis (Benjamin, 1968).

10. S. L. Passman, J. W. Nunziato and K. E. Walsh, $A$ theory of multiphase mixtures, in Rational Thermodynamics, by C. Truesdell (Springer, 1984), Appendix 5C.

11. R. Segev, Forces and the existence of stresses in invariant continuum mechanics, J. Math. Phys. 27 (1986) 163-170.

12. R. Segev, Locality and continuity in constitutive theory, Arch. Rational Mech. Anal. 101 (1988) 29-39.

13. R. Segev and G. de Botton, On the consistency conditions for force systems, Internat. J. Non-Linear Mech. 26 (1991) 47-59.

14. C. Truesdell, A First Course in Rational Continuum Mechanics (Academic Press, 1977). 